\title{
BMJ Global Health Use of clinical algorithms and rapid influenza testing to manage influenza- like illness: a cost-effectiveness analysis in Sri Lanka
}

\author{
L Gayani Tillekeratne, ${ }^{\oplus 1,2}$ Champica Bodinayake, ${ }^{3}$ Ajith Nagahawatte, ${ }^{3}$ \\ Ruvini Kurukulasooriya, ${ }^{4}$ Lori A Orlando, ${ }^{1}$ Ryan A Simmons, ${ }^{2}$ Lawrence P Park, ${ }^{1,2}$ \\ Christopher W Woods, ${ }^{1,2}$ Shelby D Reed ${ }^{1}$
}

To cite: Tillekeratne LG, Bodinayake C, Nagahawatte A, et al. Use of clinical algorithms and rapid influenza testing to manage influenza-like illness: a cost-effectiveness analysis in Sri Lanka. BMJ Glob Health 2019;4:e001291. doi:10.1136/ bmjgh-2018-001291

Handling editor Sanni Yaya

Received 8 November 2018 Revised 2 March 2019 Accepted 9 March 2019
Check for updates

(c) Author(s) (or their employer(s)) 2019. Re-use permitted under CC BY-NC. No commercial re-use. See rights and permissions. Published by BMJ.

${ }^{1}$ School of Medicine, Duke University, Durham, North Carolina, USA

${ }^{2}$ Duke Global Health Institute, Durham, North Carolina, USA

${ }^{3}$ Faculty of Medicine, University of Ruhuna, Galle, Sri Lanka ${ }^{4}$ Duke-Ruhuna Collaborative Research Centre, Galle, Sri Lanka

Correspondence to Dr L Gayani Tillekeratne; gayani.tillekeratne@duke.edu

\section{ABSTRACT}

Background Acute respiratory infections are a common reason for antibiotic overuse. We previously showed that providing Sri Lankan clinicians with positive rapid influenza test results was associated with a reduction in antibiotic prescriptions. The economic impact of influenza diagnostic strategies is unknown.

Methods We estimated the incremental cost per antibiotic prescription avoided with three diagnostic strategies versus standard care when managing Sri Lankan outpatients with influenza-like illness (ILI): (1) influenza clinical prediction tool, (2) targeted rapid influenza testing and (3) universal rapid influenza testing. We compared findings with literature-based estimates of the cost of antimicrobial resistance attributable to each antibiotic prescription.

Results Standard care was less expensive than other strategies across all parameter values in one-way sensitivity analyses. The incremental cost per antibiotic prescription avoided with clinical prediction versus standard care was US\$3.0, which was lower than the base-case estimate of the cost of antimicrobial resistance per ILI antibiotic prescription (US\$12.5). The incremental cost per antibiotic prescription avoided with targeted testing and universal testing versus standard care were both higher than the base-case cost of antimicrobial resistance per ILI antibiotic prescription: US\$49.1 and US\$138.3, respectively. To obtain a cost-effectiveness ratio lower than US\$12.5 with targeted testing versus standard care, the test price must be $<$ US\$2.6. At a higher threshold of US\$28.7, the test price must be $<$ US\$7.7.

Conclusion Clinical prediction tools and targeted rapid influenza testing may be cost-saving strategies in Sri Lanka when accounting for the societal cost of antimicrobial resistance.

\section{INTRODUCTION}

Acute respiratory tract infections (ARTIs) are a common reason for unnecessary antibiotic use worldwide. ${ }^{1-3}$ Bacterial and viral ARTIs present with similar clinical features, resulting in inappropriate treatment with antibiotics

\section{Key questions}

What is already known?

- Acute respiratory infections are a common reason for antibiotic overuse.

- Having access to rapid diagnostics tests such as rapid influenza tests may reduce inappropriate antibiotic use.

- Rapid influenza testing may not be cost-effective in resource-limited settings when only the immediate, direct costs of influenza-like illness are considered; however, no influenza diagnostic studies have accounted for the societal cost of antimicrobial resistance.

What are the new findings?

- Using targeted or universal testing with rapid influenza tests was more expensive than standard care across sensitivity analyses for outpatients in Sri Lanka.

- However, when accounting for the societal cost of antimicrobial resistance, targeted testing with rapid influenza tests was cost saving in select scenarios.

What do the new findings imply?

- Rapid influenza testing may be cost saving for managing outpatients in Sri Lanka when the societal cost of antimicrobial resistance is considered.

for the latter. ${ }^{4}$ In the USA, up to $55 \%$ of antibiotic prescriptions for ARTIs in the outpatient setting are considered to be inappropriate..$^{5-7}$ In low-income or middle-income countries (LMICs), excessive antibiotic use is even more widespread due to reasons such as limited access to diagnostic testing. ${ }^{8}$ Rapid diagnostics, which require minimal to no laboratory infrastructure and which provide a result in as little as $10 \mathrm{~min}$, are an attractive option for improving ARTI diagnosis and antibiotic use in LMICs. ${ }^{10}$ However, such diagnostics are 
generally expensive and their cost effectiveness in these settings is unknown. ${ }^{10}$

At a public tertiary care hospital in southern Sri Lanka, we previously reported that $81 \%$ of outpatients presenting with influenza-like illness (ILI) received a prescription for an antibiotic, although $64 \%$ were later identified as having viral illnesses and 39\% were identified as having influenza. ${ }^{11}$ Providing clinicians with access to rapid influenza testing was associated with a reduction in antibiotic prescriptions from $84 \%$ to $62 \%$ among patients who were influenza-positive. ${ }^{12}$

In the current analysis, we estimated the cost and cost effectiveness of managing Sri Lankan outpatients with ILI according to standard care versus the following strategies that enhance influenza diagnosis: (1) clinical prediction tool for influenza; (2) targeted rapid influenza testing of patients with a high pretest probability of influenza and (3) universal rapid influenza testing. ${ }^{13}$ Michaelidis $e t$ $a l^{14}$ previously estimated the cost of using another rapid diagnostic test, procalcitonin, for managing ARTIs in the outpatient setting in the USA. The authors performed a cost-benefit analysis of procalcitonin testing by estimating the societal cost of antimicrobial resistance per antimicrobial prescription saved, and we performed a similar analysis in Sri Lanka. ${ }^{14}$

\section{METHODS}

\section{Quasi-experimental study}

\section{Setting}

The data used to support this decision analysis model were derived from a quasi-experimental study that was performed in southern Sri Lanka from 2013 to $2015 .^{12}$ The study was conducted in the Outpatient Department (OPD) of the largest (1500 bed), public tertiary care hospital in the Southern Province of Sri Lanka. The OPD cares for approximately 1000 patients daily at no charge to patients. The population triaged to the OPD consists of patients with less severe illness who can generally be managed as outpatients; patients with more severe illness are directed to the hospital's Emergency Treatment Unit. All medications prescribed through the OPD are dispensed free of charge through the outpatient pharmacy at the hospital. Physicians in the OPD are not allowed to prescribe oseltamivir (Rs 338.4/US\$2.2 per capsule), and patients who are deemed to require antiviral therapy are admitted to receive such therapy in the inpatient setting. ${ }^{15}$

\section{Design}

The quasi-experimental study is further described in another publication. ${ }^{12}$ Briefly, 571 consecutive outpatients meeting the WHO's definition of ILI (fever $\geq 38^{\circ} \mathrm{C}$ and cough in the past 7 days) and presenting to the OPD were enrolled from March 2013 to January 2015. ${ }^{16}$ Clinical information and two nasal or nasopharyngeal samples were obtained from each patient: one for multiplex PCR testing for respiratory pathogens and one for rapid influenza testing using an antigen-based test for influenza A/B. ${ }^{11}$ The samples for rapid influenza testing were tested immediately using the Veritor Flu A+B system (Becton, Dickinson and Company, Franklin Lakes, New Jersey, USA), with the result available within $15 \mathrm{~min}$, and the test result was only released to physicians during the second half of the study (Phase II). Physicians were provided with the results from rapid influenza testing prior to any clinical decision-making, including the prescription of antimicrobials, during Phase II. For confirmation of influenza, the second nasopharyngeal sample was tested retrospectively using real-time reverse transcription PCR (RT-PCR) with the Luminex xTAG respiratory virus panel (Luminex Molecular Diagnostics, Toronto, Canada). During phase I when physicians were not provided with results, $46.5 \%$ of patients tested positive for influenza by rapid test, while during phase II, $28.6 \%$ of patients tested positive for influenza by rapid test. Seasonal and annual variation in influenza likely accounted for differences in influenza positivity between the two phases. ${ }^{11}$ Of patients who were influenza positive, $83.7 \%$ received antibiotics in phase I and $62.3 \%$ received antibiotics in phase II. ${ }^{12}$ Of patients who were influenza negative, $79.3 \%$ received antibiotics in phase I and $72.5 \%$ received antibiotics in phase II.

\section{Patient and public involvement}

Patients were not directly involved in this study, as this analysis was conducted using data derived from a previously published quasi-experimental study. ${ }^{12}$

\section{Model structure}

We evaluated the cost of managing ILI among outpatients in Sri Lanka by examining four models of care (figure 1): (1) standard care-usual care with no access to clinical prediction tools or rapid influenza testing; (2) clinical prediction-using a clinical prediction tool that we previously developed to identify patients with a higher probability of influenza; (3) targeted testing-rapid influenza testing of outpatients with a higher pretest probability of influenza per the clinical prediction tool and (4) universal testing-rapid influenza testing of all outpatients. ${ }^{13}$ An ILI treatment episode was used as the time horizon in the base-case analysis. Decision analysis modelling was performed using TreeAgePro 2017 V.2017 (TreeAge Software, Williamstown, Massachusetts, USA).

\section{Probabilities}

Probabilities representing each management strategy in the decision analysis model were based on data from the quasi-experimental trial. Depending on the strategy, probabilities were calculated from phase I of the study, phase II of the study or the entire study (table 1). Probabilities were derived from the primary data set of 567 patients who had both a definitive influenza PCR test result and a definitive influenza rapid test result and thus may differ minimally from previously published values. 


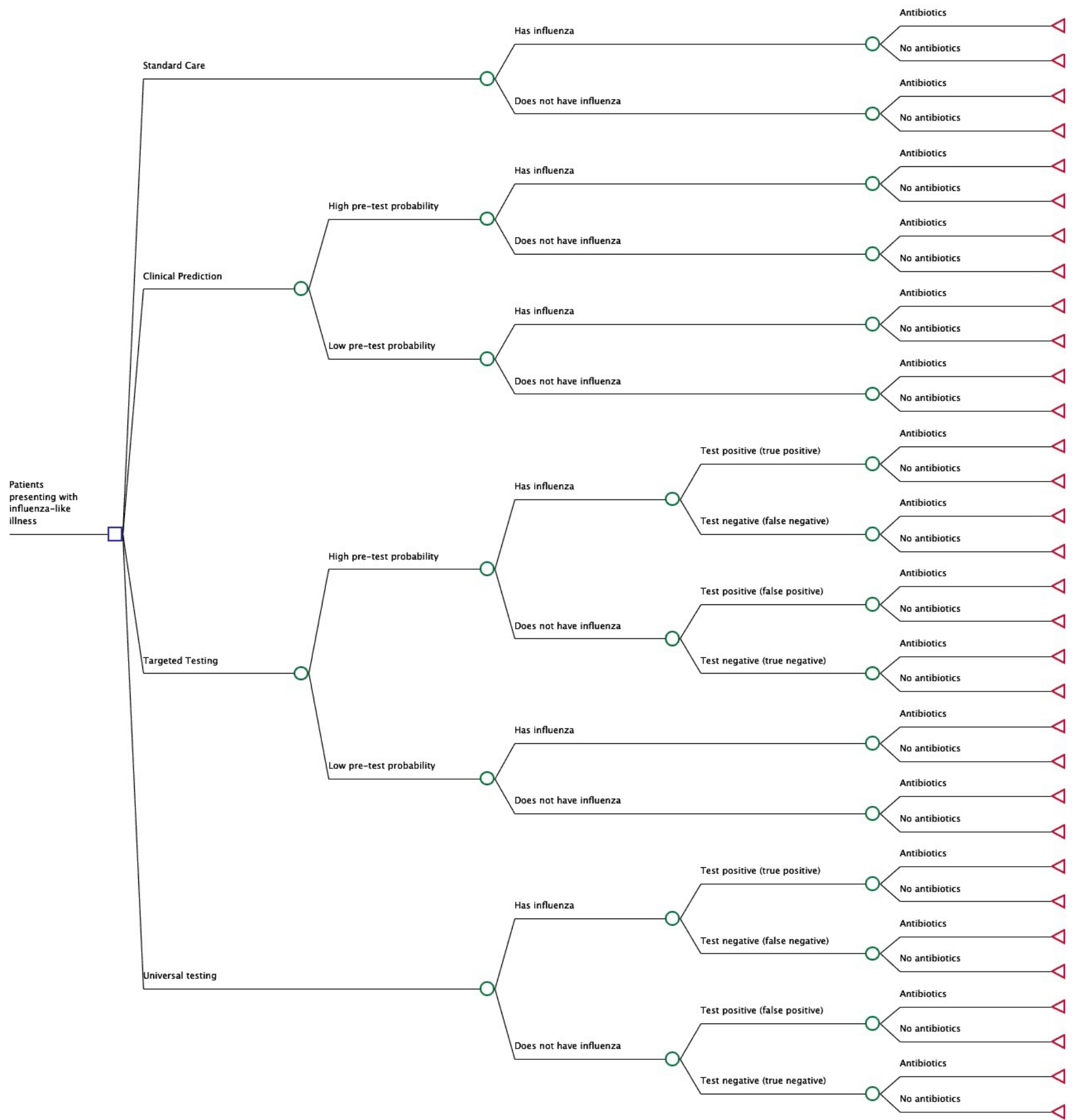

Figure 1 Decision analysis tree with diagnostic strategies for outpatients with influenza-like illness in southern Sri Lanka. Probabilities used are presented in table 1.

\section{Inputs to estimate costs of ILI management strategies}

To determine the cost of antibiotics, we assumed that a usual course of antibiotics consisted of amoxicillin $500 \mathrm{mg}$ two times a day for 3 days. Prior data indicate that penicillins are the most common type of antibiotic prescribed for ARTIs in the OPD and that most prescriptions are dispensed for a duration of 3 days. ${ }^{12} 17$ The price for purchasing $500 \mathrm{mg}$ capsules through the Health Ministry in Sri Lanka was Rs 4.40 (US\$0.03) per capsule or Rs 26.40 (US\$0.17) per course in 2017 (US\$1 =Rs 151.7; table 2). ${ }^{18}$ To estimate the cost of physicians' time, it was assumed that using the clinical prediction algorithm to determine patients who were at low or high risk for influenza would take 5 min of physicians' time, that counselling about the decision to withhold antibiotics would take $5 \mathrm{~min}$ of physicians' time for patients in the clinical algorithm arm, and that testing using the rapid influenza test kit would take 20 


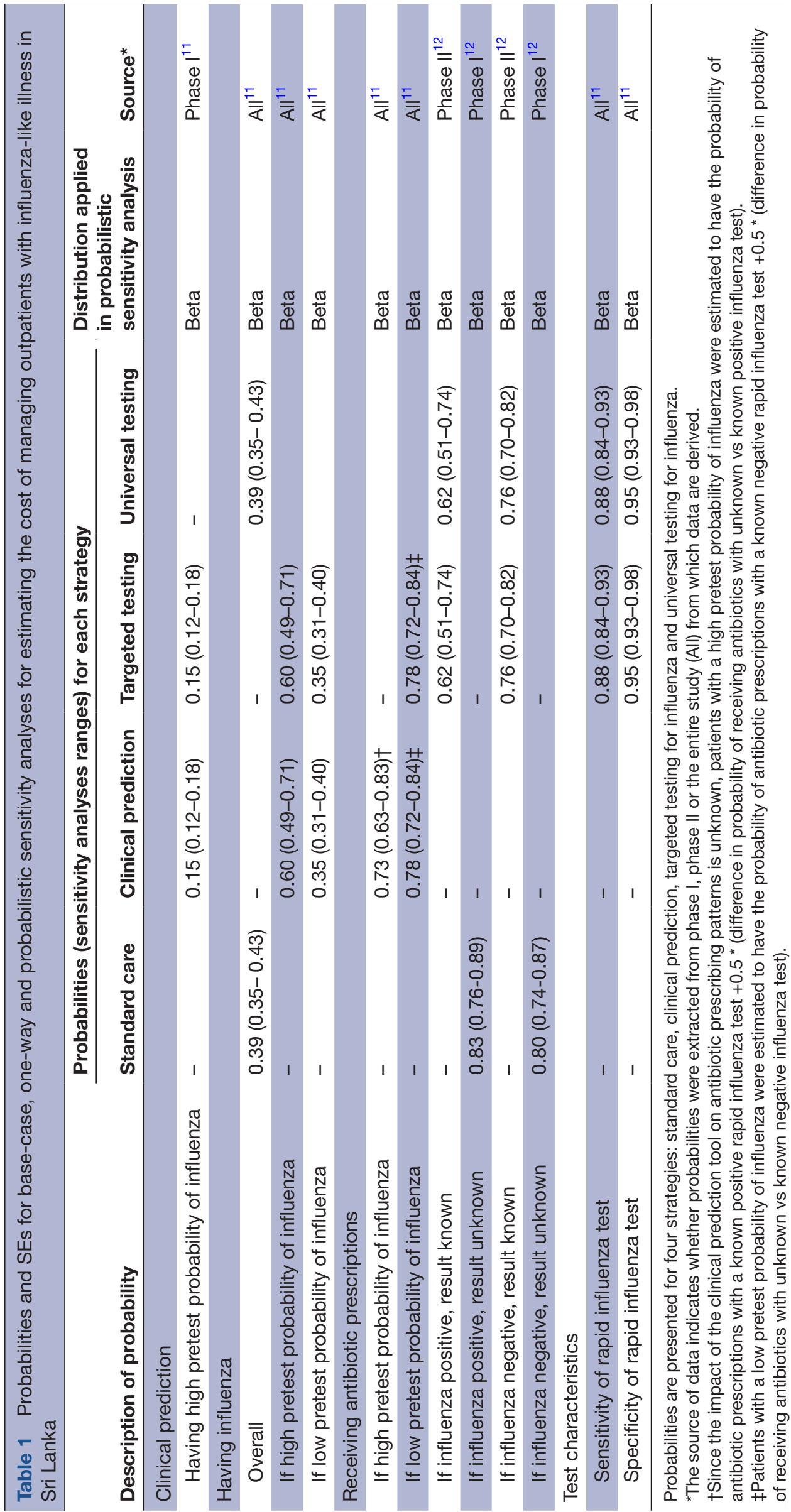


Table 2 Costs for base case, low and high estimates of managing outpatients with ARTIs in Sri Lanka

\begin{tabular}{llll}
\hline Costs & $\begin{array}{l}\text { Base } \\
\text { case }\end{array}$ & Low & High \\
\hline Antibiotics & $\$ 0.17$ & $\$ 0.02$ & $\$ 1.70$ \\
$\begin{array}{l}\text { Rapid influenza test } \\
\begin{array}{l}\text { Physician time for using clinical } \\
\text { algorithm }\end{array}\end{array}$ & $\$ 14.00$ & $\$ 1.40$ & $\$ 140.00$ \\
$\begin{array}{l}\text { Physician time for counselling } \\
\text { about withholding antibiotics }\end{array}$ & $\$ 0.10$ & $\$ 0.01$ & $\$ 1.00$ \\
$\begin{array}{l}\text { Physician time for rapid } \\
\text { influenza testing }\end{array}$ & $\$ 0.40$ & $\$ 0.04$ & $\$ 1.00$ \\
\hline
\end{tabular}

min of physicians' time. The monthly salary for Grade 2 medical officers was estimated at Rs 28095 per month in 2014 , which adjusts to US\$208.64 per month or US\$0.02 per minute. ${ }^{20}$ To determine the cost of the Becton Dickinson Veritor rapid influenza test, it was estimated that the small portable reader, which can process 3000 tests before needing to be replaced, cost US $\$ 300 .^{21}$ A box of 30 test kits with appropriate controls is estimated to cost US $\$ 416$. $^{22}$ Thus, accounting for the cost of the testing device, it was estimated that one rapid influenza test would cost US $\$ 14$. To account for uncertainty in estimates, we allowed for wide variation in low and high estimates by adjusting by 0.1 or 10 times the base-case cost, respectively.

\section{Base-case cost-effectiveness analysis}

Since the OPD generally treats patients with less severe illness who can be managed as outpatients, we assumed that there would be no difference across management strategies with regard to immediate health outcomes such as treatment-related side effects, hospitalisation and death. Reductions in antibiotic prescribing were used to measure the incremental effectiveness of each of the three management strategies versus standard care. Common practice in cost-effectiveness analysis requires ordering management strategies by increasing cost, identifying dominated (ie, most costly, less effective) strategies and estimating incremental costs and effectiveness of increasingly costly strategies compared with less costly strategies that meet acceptable cost-effectiveness thresholds. We deviated from this approach by comparing all three management strategies (ie, clinical prediction, targeted testing and universal testing) to standard care because there is no threshold in Sri Lanka on which to judge the acceptability of an incremental cost per antibiotic prescription saved. Also, use of standard care as the common comparator directly generates the per-patient budgetary impact of implementing each of these strategies in the OPD rather than incremental costs between management strategies. None of the alternative management strategies is used routinely in Sri Lanka.

\section{Estimated cost of antibiotic resistance per antibiotic prescribed}

As acknowledged above, the maximum acceptable cost in Sri Lanka to reduce the use of antibiotics is unknown. To provide more context to the cost-effectiveness ratios representing the incremental cost per antibiotic prescription avoided, we estimated the societal cost of antibiotic resistance associated with each antibiotic prescription.

The societal direct cost of antibiotic resistance has not been estimated for most countries in the world, including for Sri Lanka. To generate an estimate for Sri Lanka, we adapted a study from the US setting in which the direct societal cost was estimated at US\$20 billion annually (2008 dollars). ${ }^{23}{ }^{24}$ We converted US\$ to Sri Lankan Rupees using the purchasing power parity (PPP) exchange rate, which is the number of units of a country's currency required to buy the same amount of goods and services in a country as the US\$ would buy in the USA. ${ }^{25}$ Using 2017 values of a PPP exchange rate of 48.5 Sri Lankan rupees per US\$, a population of 21444 000 in Sri Lanka and a population of 325719178 in the USA, the direct cost of antibiotic resistance in Sri Lanka adjusted for the population would be 63.9 billion rupees or US\$420 $967552 .{ }^{26-28}$ We also estimated the societal cost of antibiotic resistance in Sri Lanka by adapting a study from Thailand. In Thailand, the direct cost of antimicrobial resistance is estimated to be between US $\$ 70$ and US\$170 million. ${ }^{29} 30$ Using a midpoint value of US\$120 million and a population of 69037513 in Thailand in 2017, the direct cost of antibiotic resistance in Sri Lanka adjusted for the population would thus be US\$37 $273649 .{ }^{31}$ Given the wide variation between the estimate from the US study (61.4 US\$ per person in the USA) and the Thai estimate (US $\$ 1.7$ per person in Thailand), we used the midpoint value of US\$229 120601 (US\$10.7 per person in Sri Lanka) as the base-case estimate of the societal cost of antibiotic resistance in Sri Lanka in this analysis (table 3 ).

The relationship between antibiotic use and antibiotic resistance is not well understood. For this analysis, we assumed a one-to-one linear relationship between the two, as assumed by Michaelidis et al. ${ }^{14}$ To determine the impact of antibiotics used in treating ILI on overall societal antibiotic resistance, the proportion of antibiotics used for humans versus animals is necessary. In the USA, it is estimated that up to $80 \%$ of all antibiotics are used in the agricultural/animal sector; for Sri Lanka and much of the rest of the world, this proportion is unknown. ${ }^{32}$ In Sri Lanka, it is estimated that the majority of antibiotics used in animal feed is used for poultry, pigs and cattle. ${ }^{32} 33$ In 2011, it was estimated that there were 1.4 million cattle, 80000 pigs and 15.4 million poultry in Sri Lanka or 16.9 million livestock total and 0.83 livestock: human. ${ }^{33}$ In the USA, it was estimated that there were 90.1 million cattle and 66.3 million pigs in 2013 and 8.5 billion broiler heads in 2014 or 8.7 billion livestock total and 27.1 livestock: human. ${ }^{35}$ Using the US estimate of $80 \%$ of antibiotics 
Table 3 Estimated direct societal cost of antibiotic resistance per antibiotic prescribed for the outpatient management of ARTIs in Sri Lanka, estimated in US\$

\begin{tabular}{|c|c|c|c|c|c|}
\hline Row & Inputs and outputs & Base case & Low & High & Source \\
\hline \multicolumn{6}{|l|}{ Input } \\
\hline a & Annual total cost of antibiotic resistance & $\$ 229120600$ & $\$ 114560300$ & $\$ 343680900$ & $143467-69$ \\
\hline $\mathrm{C}$ & $\begin{array}{l}\text { Impact of human versus animal antibiotic } \\
\text { use on societal cost of resistance }\end{array}$ & $200 \%$ * & $100 \%$ & $300 \%$ & 14 \\
\hline $\mathrm{e}$ & Outpatient care occurring in public sector & $40 \%$ & $20 \%$ & $60 \%$ & 39 \\
\hline$f$ & $\begin{array}{l}\text { Percentage of outpatient visits that are for } \\
\text { ARTIs }\end{array}$ & $10.5 \%$ & $5.3 \%$ & $15.8 \%$ & 40 \\
\hline$g$ & Annual antibiotic prescriptions & 35920825 & 35920825 & 3592025 & 36 \\
\hline
\end{tabular}

*Estimate from Michaelidis et $a 1^{14}$ used given lack of data for Sri Lanka. ARTIs, acute respiratory tract infections.

being used in livestock and scaling for livestock, it is estimated that $2.4 \%$ of antibiotics in Sri Lanka are used for livestock.

For annual antibiotic prescriptions, it was estimated that 10470 standard units (pill, capsule or ampoule) per 1000 population were used in 2013 in Sri Lanka. ${ }^{36}$ If it is assumed that six standard units comprise one prescription (ie, two capsules of $500 \mathrm{mg}$ amoxicillin daily for 3 days, which is a standard antibiotic prescription given through the OPD), then 35920825 antibiotic prescriptions were dispensed in 2013. The percentage of antibiotics used in the outpatient sector in Sri Lanka is unknown; since overall outpatient antibiotic use in other countries generally far exceeds inpatient antibiotic use (reported ranges of $80 \%-90 \%$ ), we assumed that $80 \%$ of antibiotics were prescribed in the outpatient setting, as used in the analysis by Michaelidis et al. ${ }^{143738}$ We used published estimates for the percentage of outpatient care in Sri Lanka occurring in the public sector (40\%) and the percentage of outpatient visits that are for ARTIs $(10.5 \%) .{ }^{39} 40$

To generate low and high estimates of the cost of antimicrobial resistance per additional antibiotic prescribed, we varied all inputs by a factor of 0.5 or 1.5 times the basecase values with the exception of the annual number of antibiotic prescriptions (table 3).

All costs in this analysis were in 2017 US\$ unless otherwise stated. Costs in Sri Lankan rupees were adjusted by multiplying by annual inflation rates in Sri Lanka and then adjusting for US dollars (US\$1=Rs 151.7). ${ }^{1841}$

\section{Sensitivity analysis}

Multiple one-way sensitivity analyses were performed to evaluate the extent to which findings form the decision model changed when specific inputs and assumptions were varied (ranges used for probabilities and costs are listed in tables 1 and 2, respectively). In probabilistic sensitivity analyses, all probabilities in the model were varied simultaneously over specified beta distributions while published unit costs were treated as constant to isolate the impact of uncertainty stemming from the clinical study. We also performed threshold analyses in which we varied costs and probabilities to the point at which the cost per antibiotic prescription saved with the three strategies became lower than the low, base-case and high estimated costs of antimicrobial resistance per additional antibiotic prescribed.

\section{RESULTS}

\section{Costs and cost-effectiveness analyses}

In the base-case scenario, the estimated cost per patient managed in the standard care arm (US\$0.14) was lower than the per-patient cost in the clinical prediction (US\$0.25), targeted testing (US\$2.41) and universal testing (US\$14.52) arms (table 4). The number of prescriptions per patient was highest in the standard care arm (0.81) compared with the other three management strategies: clinical prediction (0.77), targeted testing (0.77) and universal testing (0.71). The incremental cost-effectiveness ratio (ICER) per antibiotic prescription 
Table 4 Estimated costs and cost effectiveness of management strategies versus standard care: base-case analysis

\begin{tabular}{lllll} 
& Standard care & Clinical prediction & Targeted testing & Universal testing \\
\hline Cost per patient (US\$) & $\$ 0.14$ & $\$ 0.25$ & $\$ 2.41$ & $\$ 14.52$ \\
Number of antibiotics per patient & 0.81 & 0.77 & 0.77 & 0.71 \\
$\begin{array}{l}\text { Incremental cost per antibiotic prescription } \\
\text { avoided (vs standard care) }\end{array}$ & $\mathrm{NA}$ & $\$ 3.0$ & $\$ 49.1$ & $\$ 138.3$ \\
\hline
\end{tabular}

safely avoided was US\$3.0 for clinical prediction, US $\$ 49.1$ for targeted testing and US $\$ 138.3$ for universal testing.

In one-way sensitivity analyses, the number of prescriptions was always highest in the standard care arm except when the probability of an antibiotic prescription with a low pretest probability of influenza was greater than $84 \%$, when both clinical prediction and targeted testing had a higher number of prescriptions. The number of prescriptions was lowest in the universal testing arm across all parameters tested in the one-way sensitivity analyses. In one-way sensitivity analyses, standard care was always the least costly strategy. No strategies were clearly dominated when compared with standard care.

\section{Comparing ICERs with estimated cost of antimicrobial resistance per antibiotic prescription}

The base-case cost of antimicrobial resistance per antibiotic prescribed was estimated as US $\$ 12.5$ per antibiotic prescription (table 3). When comparing the estimated ICERs of the three ILI management strategies versus standard care, only the ICER for the clinical prediction strategy was less than US\$12.5

Threshold analyses were conducted to determine values for probabilities and costs at which the incremental cost per antibiotic prescription avoided in the Clinical Prediction, Targeted Testing or Universal Testing arms compared with Standard Care would be less than the estimated low, base-case and high costs of antibiotic resistance per ILI prescription (US $\$ 1.6$, US $\$ 12.5$ and US\$28.7, respectively; figure 2). In the base-case cost and probability scenarios, the ICER of clinical prediction compared with standard care was always less than the base and high costs of antibiotic resistance per ILI prescription avoided. In the base-case cost and probability scenarios, the ICER of the two testing strategies compared with Standard Care well surpassed the estimated cost of antimicrobial resistance per prescription avoided. The ICER of Targeted Testing compared with Standard Care was less than the high estimated cost of antibiotic resistance per ILI prescription (US\$28.7) when the cost of the rapid influenza test was less than US\$7.7, and the ICER was less than the base-case estimated cost of antibiotic resistance per ILI prescription (US\$12.5) when the cost of the rapid influenza test was less than US\$2.6. The ICER of universal testing compared with standard care was less than US\$28.7 (the high estimated cost of antibiotic resistance per ILI prescription) when the cost of the rapid influenza test was less than US\$2.6.
In probabilistic sensitivity analyses, the ICERs for clinical prediction versus standard care were less than US $\$ 12.5$ in $72.0 \%$ of simulations and less than US\$1.6 in $39.0 \%$ of simulations (figure 3). The ICERs for both testing strategies surpassed even the high estimate of the cost of antimicrobial resistance of US\$28.7, and this finding held across all simulations in the probabilistic sensitivity analyses.

\section{DISCUSSION}

We determined the cost and cost benefit of using a clinical prediction tool and rapid influenza testing to manage outpatients with ILI in Sri Lanka. We found that when considering direct costs alone, strategies employing clinical prediction tools and rapid influenza testing were more costly compared with standard care. However, when taking the societal cost of antimicrobial resistance into account in a cost-benefit analysis, using a clinical prediction tool was less costly and more effective than standard care across all base-case probabilities and costs, and targeted rapid influenza testing was less costly and more effective in select scenarios.

Studies exploring ILI and ARTI diagnostic strategies from a purely cost-effectiveness standpoint have generally shown that diagnostic strategies only appear to be cost effective when accounting for serious outcomes such as hospitalisation. For example, You et a $t^{42}$ found that a molecular point-of-care test for influenza was cost effective compared with clinical judgement among elderly outpatients presenting with ILI during influenza season in Hong Kong, when accounting for hospitalisations and mortality reduced in estimating QALYs saved with diagnostic testing. In addition, cost-effectiveness analyses of diagnostic testing compared with treatment strategies have shown that empiric treatment with antivirals in the outpatient setting are often more cost effective than using diagnostic testing to guide decision-making. ${ }^{43-45}$ However, such studies have not accounted for the cost of inappropriate antibiotic use and the wider societal cost of antimicrobial resistance. Michaelidis $e t \mathrm{al}^{14}$ performed one of the first such analyses and showed that procalcitonin testing could be cost beneficial when the societal cost of antimicrobial resistance was considered and testing was limited to adults with ARTIs judged by their physicians to require antibiotics. More recently, Lubell et $a l^{46}$ showed that in the Vietnamese setting, C reactive protein testing could be cost beneficial when the societal 
A

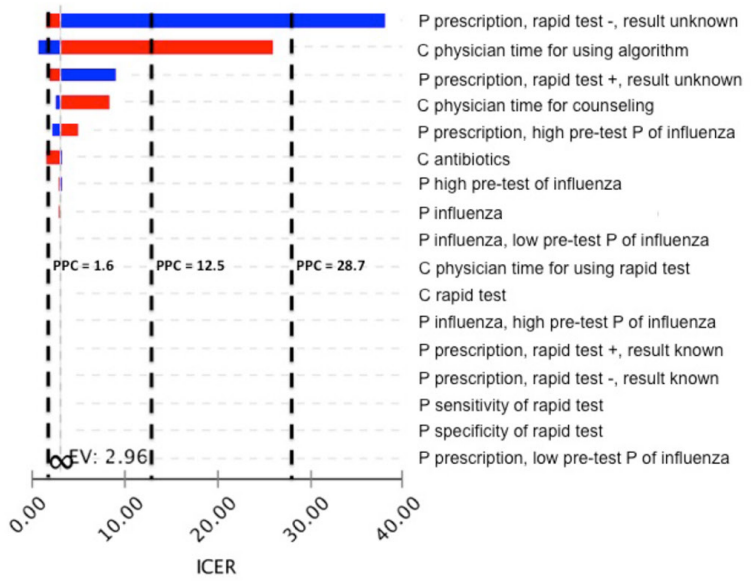

B

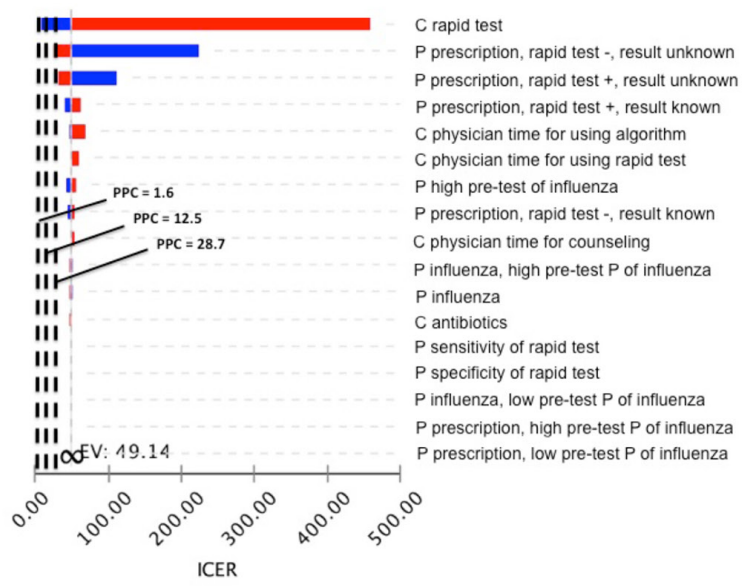

C

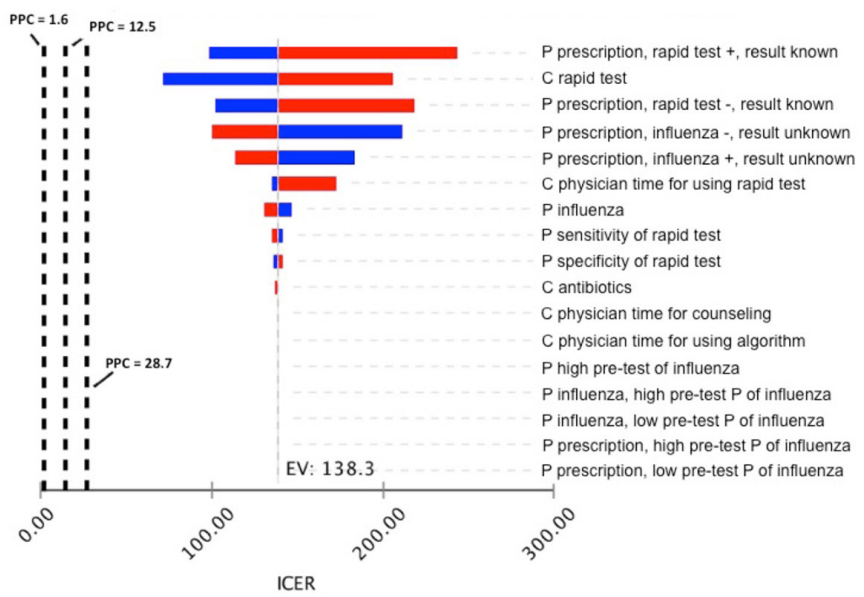

Figure 2 (A) Clinical prediction versus standard care. (B) Targeted testing versus standard care. (C) Universal versus standard care.

cost of antimicrobial resistance was considered, but only when physicians' adherence to test results was higher than the $64 \%$ demonstrated in the clinical trial setting. Our findings using methods similar to these prior two studies show that targeted rapid influenza testing could be cost beneficial in select scenarios in Sri Lanka. Our results showing a per-prescription cost of antimicrobial resistance ranging from US $\$ 1.6$ to US $\$ 26.8$ are similar to estimates by Shrestha et at $t^{47}$ showing that the cost of antimicrobial resistance associated with a course of amoxicillin-clavulanate in Thailand ranges from US $\$ 2.9$ to US\$32.2.

Given recent estimates showing that global antimicrobial resistance could result in cumulative losses of up to US $\$ 100$ trillion by 2050 , accounting for the economic cost of antimicrobial resistance in cost-benefit studies is important. ${ }^{48}$ In Sri Lanka, healthcare is available free of charge to all patients and incorporating societal economic measures into policy decisions may be even more compelling. Studies indicate that both antimicrobial resistance and antimicrobial overuse are prevalent in Sri Lanka, highlighting the need for new approaches that can counteract these inter-related problems. ${ }^{49} 50$

Strategies that employ diagnostics are inherently more expensive, thus have to provide reasonable benefit in order to be considered cost effective or cost beneficial. In our study, the cost of the rapid diagnostic was several magnitudes greater than the cost of physician time or antibiotics. Accounting for hidden costs of antibiotic resistance may enhance the appeal of diagnostics-based strategies and may reveal that traditional treatment-focused strategies are ultimately more costly from a societal perspective. For instance, Oppong et $a l^{51}$ recently showed that the empiric treatment of lower respiratory tract infections with amoxicillin in Europe was no longer cost effective when taking into account the societal cost of antimicrobial resistance. While accounting for hidden costs may increase tolerance for more expensive diagnostics, low-cost tests are ultimately needed for lower-resource settings seeking to implement diagnostic testing. ${ }^{52}$ At present, commercially available rapid influenza diagnostic tests remain in the range of US\$10-20 or greater and are likely not affordable in most LMIC settings. ${ }^{42} 5354$

In concert with increased availability of lower-priced diagnostics, strategies that enhance the impact of diagnostics are critically needed. In our study, providing results from the rapid diagnostic was associated with a modest absolute decrease of $\sim 20 \%$ in antibiotic prescriptions among patients with a positive rapid influenza test result. Even in randomised controlled studies in the USA and Europe, rapid influenza testing has resulted in an absolute difference of $20 \%-30 \%$ in antibiotic prescriptions between patients who were influenza-positive versus influenza-negative, although the baseline prevalence of antibiotic prescriptions was lower. ${ }^{55} 56$ Since adherence to test results shows wide regional variation-for instance, physicians in Europe generally follow procalcitonin results much more stringently than physicians in the USA-local educational strategies may need to be employed. ${ }^{57-59}$ Approaches that modify physician behaviour, such as public reporting of prescription rates to attain a higher level of transparency and accountability, may provide both financial and reputational incentives 


\section{CE Acceptability Curve}

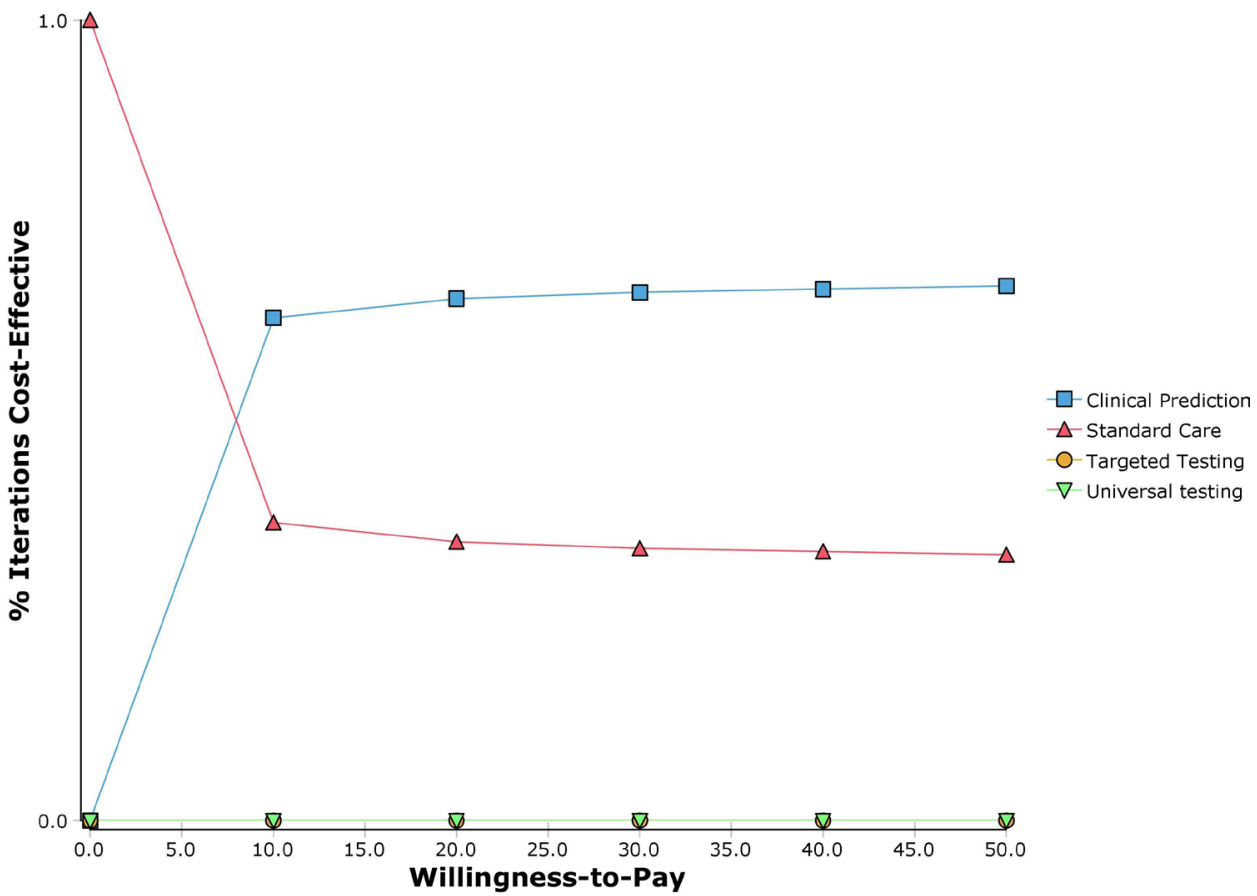

Figure 3 Probabilistic sensitivity analysis of clinical prediction, targeted testing and universal testing versus standard care for outpatient management of influenza-like illness in Sri Lanka. Results are presented in the form of an acceptability curve, with the $\mathrm{x}$-axis showing willingness-to-pay thresholds or per-prescription costs of antimicrobial resistance (in US\$) and the y-axis showing the likelihood that strategies would be considered cost-effective for each willingness-to-pay threshold.

to improve quality of care.$^{60}$ Reporting to patients may result in little effect, as we have previously shown that health literacy regarding antimicrobials in this setting is relatively low. ${ }^{17}$ However, reporting to colleagues may be effective since physicians in Sri Lanka pass through an educational system that relies on a public, national ranking system and physicians may be accustomed to an open, competitive system. Reporting to administration may also be effective since the Sri Lanka Ministry of Health employs all physicians in the public sector; however, such reporting would have to be conducted in a non-punitive fashion that results in positive change. Innovative techniques such as pay-for-performance or provider payment as an incentive to decrease antimicrobial prescriptions may also have a role in this setting and may ultimately be less expensive and more cost effective than using advanced diagnostics. ${ }^{60-62}$

The strengths of this study include the use of data generated from a prior quasi-experimental study in the same hospital setting to estimate probabilities. Therefore, our analyses may more closely approximate real-world numbers and be more generalisable than results from randomised controlled trials. In addition, we selected wide ranges for cost and probability estimates to account for uncertainty in estimates.

Several limitations must be noted. Our population was comprised of patients with ILI and thus our results may not be generalisable to patients with general ARTIs. The cost of antibiotic resistance from a societal perspective was estimated using a series of assumptions since such data are not available but critically needed for Sri Lanka. We laid out each assumption in a transparent fashion such that our estimates could be reproduced, and we also used wide ranges in parameters to account for uncertainty in estimates. The societal cost of antimicrobial resistance in Sri Lanka was extrapolated using estimates from the USA and Thailand. Even for the USA, the widely quoted estimate of 20 billion US\$ in direct costs was based on results from one tertiary care hospital comparing the cost of antibiotic-resistant infections compared with other hospitalised patients. ${ }^{24}$ More recent estimates by Thorpe et $a l^{63}$ estimate the total direct cost as 2.2 billion US\$ when compared with antibiotic-susceptible infections. The cost of antimicrobial resistance is often omitted from analyses, partly due to the challenges associated with quantifying this cost; however, the need for such estimates is great and others have started estimating this cost using methods similar to ours, with results that are in similar ranges. ${ }^{46}{ }^{47}$ We did not assess the impact of oseltamivir use in this population, since the antiviral is not prescribed through the OPD and the patient population did not have severe disease. Our results are thus generalisable to other OPD populations with similar disease severity. We estimated the impact of using the clinical algorithm on reducing antibiotic prescriptions, since this tool has not been employed in clinical practice. We chose to use a linear relationship between antimicrobial consumption and antimicrobial resistance, as previously assumed by Michaelidis $e t a l,{ }^{14}$ since this relationship is unknown. Several studies have explored the complex 
correlation between antimicrobial consumption and resistance, with widely varying estimates. ${ }^{476465}$ An almost linear relationship was shown in ecological analyses between macrolides and macrolide-resistant Streptococcus pneumoniae by Albrich et al. ${ }^{66}$ Finally, the prescription of antimicrobials decreased between the two phases of the original quasi-experimental study; the decrease in antibiotic prescriptions in the second phase may thus have been independent of the influenza rapid test result. However, the decrease in antimicrobial prescriptions was significantly greater in patients who were influenza-positive compared with patients who were influenza-negative. The limitations associated with interpreting quasi-experimental study results were further described in the original publication. ${ }^{12}$

In conclusion, we found that managing outpatients with ILI in Sri Lanka using clinical algorithms or targeted rapid influenza testing may be less costly than standard care when the societal cost of antimicrobial resistance is considered. Our analyses provide important preliminary estimates regarding the economic impact of using rapid diagnostics for ILI in Sri Lanka.

Acknowledgements The authors acknowledge support provided by the Duke Global Health Institute and the Hubert-Yeargan Center for Global Health.

Contributors LGT conceived the study, performed the analyses and drafted the manuscript. $\mathrm{CB}, \mathrm{AN}$ and RK helped with data acquisition and revising the manuscript. LA0, RAS and LPP helped with data analyses and revising the manuscript. CWW and SDR helped with study design, interpretation of findings and revising the manuscript

Funding LGT was supported by supported by a grant from the National Institutes of Allergy and Infectious Diseases (K23Al125677).

Competing interests CWW has served in an advisory capacity to Becton, Dickinson, and Company, who provided the rapid influenza tests used in the study from which data were derived.

Patient consent for publication Not required.

Provenance and peer review Not commissioned; externally peer reviewed.

Data availability statement All data used in these analyses have been provided in detail in the text.

Open access This is an open access article distributed in accordance with the Creative Commons Attribution Non Commercial (CC BY-NC 4.0) license, which permits others to distribute, remix, adapt, build upon this work non-commercially, and license their derivative works on different terms, provided the original work is properly cited, appropriate credit is given, any changes made indicated, and the use is non-commercial. See: http://creativecommons.org/licenses/by-nc/4.0/.

\section{REFERENCES}

1. Grijalva CG, Nuorti JP, Griffin MR. Antibiotic prescription rates for acute respiratory tract infections in US ambulatory settings. JAMA 2009;302:758-66.

2. Magill SS, Edwards JR, Beldavs ZG, et al. Prevalence of antimicrobial use in US acute care hospitals, May-September 2011. JAMA 2014;312:1438-46.

3. Laxminarayan R, Duse A, Wattal C, et al. Antibiotic resistance-the need for global solutions. Lancet Infect Dis 2013;13:1057-98.

4. Zaas AK, Garner BH, Tsalik EL, et al. The current epidemiology and clinical decisions surrounding acute respiratory infections. Trends Mol Med 2014;20:579-88.

5. Donnelly JP, Baddley JW, Wang HE. Antibiotic utilization for acute respiratory tract infections in U.S. emergency departments. Antimicrob Agents Chemother 2014;58:1451-7.

6. Fleming-Dutra KE, Hersh AL, Shapiro DJ, et al. Prevalence of inappropriate antibiotic prescriptions among US ambulatory care visits, 2010-2011. JAMA 2016;315:1864-73.
7. Shapiro DJ, Hicks LA, Pavia AT, et al. Antibiotic prescribing for adults in ambulatory care in the USA, 2007-09. J Antimicrob Chemother 2014;69:234-40.

8. Bhavnani D, Phatinawin L, Chantra S, et al. The influence of rapid influenza diagnostic testing on antibiotic prescribing patterns in rural Thailand. Int J Infect Dis 2007;11:355-9.

9. Ozkaya E, Cambaz N, Coskun Y, et al. The effect of rapid diagnostic testing for influenza on the reduction of antibiotic use in paediatric emergency department. Acta Paediatr 2009;98:1589-92.

10. Zumla A, Al-Tawfiq JA, Enne VI, et al. Rapid point of care diagnostic tests for viral and bacterial respiratory tract infections-needs, advances, and future prospects. Lancet Infect Dis 2014;14:1123-35

11. Shapiro D, Bodinayake CK, Nagahawatte A, et al. Burden and seasonality of viral acute respiratory tract infections among outpatients in southern Sri Lanka. Am J Trop Med Hyg 2017;97:88-96.

12. Tillekeratne LG, Bodinayake CK, Nagahawatte A, et al. Use of rapid influenza testing to reduce antibiotic prescriptions among outpatients with influenza-like illness in southern Sri Lanka. Am J Trop Med Hyg 2015;93:1031-7.

13. Tillekeratne LG, Bodinayake CK, Nagahawatte A, et al. An underrecognized influenza epidemic identified by rapid influenza testing, southern Sri Lanka, 2013. Am J Trop Med Hyg 2015;92:1023-9.

14. Michaelidis $\mathrm{Cl}$, Zimmerman RK, Nowalk MP, et al. Cost-effectiveness of procalcitonin-guided antibiotic therapy for outpatient management of acute respiratory tract infections in adults. J Gen Intern Med 2014;29:579-86.

15. Lanka SPCoS. Pharmaceutical items, 2017. Available: http://www. spc.lk/pub/spc-awarded-tenders-pharmaceutical-2017-january.xls [Accessed 6 Jan 2019].

16. World Health Organization. Who interim global epidemiological surveillance standards for influenza; 2012.

17. Tillekeratne LG, Bodinayake CK, Dabrera T, et al. Antibiotic overuse for acute respiratory tract infections in Sri Lanka: a qualitative study of outpatients and their physicians. BMC Fam Pract 2017;18.

18. Central Bank of Sri Lanka. Current economic indicators, 2010. Available: http://www.cbsl.gov.lk/htm/english/_cei/er/e_1.asp [Accessed 5 Jan 2018].

19. State Pharmaceuticals Corporation. State pharmaceuticals Corporation price list, 2017. Available: http://www.spc.Ik/pub/pricelist.pdf [Accessed 5 Jan 2018].

20. Public Service Commission. The Gazette of the Democratic socialist Republic of Sri Lanka; 2014.

21. 4MD Medical Solutions. BD Veritor System reader, 2017. Available: https://www.4mdmedical.com/bd-veritor-system-reader-forinfluenza-a-b-rsv-kits.html [Accessed 24 Nov 2017].

22. 4MD Medical Solutions. BD Veritor System influenza A+B POC kit, 2017. Available: https://www.4mdmedical.com/laboratory/point-ofcare/rapid-tests/influenza/bd-veritor-system-influenza-a-b-poc-kit. html [Accessed 24 Nov 2017].

23. Centers for Disease Control and Prevention. Antibiotic Resistance Threats in the United States, 2013, 2013.

24. Roberts RR, Hota B, Ahmad I, et al. Hospital and societal costs of antimicrobial-resistant infections in a Chicago teaching hospital: implications for antibiotic stewardship. Clin Infect Dis 2009;49:1175-84.

25. World Health Organization. Purchasing power parity 2005, 2017. Available: http://www.who.int/choice/costs/ppp/en/ [Accessed 23 Nov 2017].

26. International Monetary Fund. Implied ppp conversion rate, 2017. Available: http://www.imf.org/external/datamapper/PPPEX@WEO/ OEMDC/ADVEC/WEOWORLD [Accessed 5 Jan 2018]

27. Department of Census and Statistics SL. Population and housing, 2017. Available: http://www.statistics.gov.lk/page.asp?page= Population and Housing [Accessed 5 Jan 2018].

28. United States Census Bureau. National population Totals and components of change, 2017. Available: https://www.census.gov/ data/tables/2017/demo/popest/nation-total.html [Accessed 5 Jan 2018].

29. Sumpradit N, Wongkongkathep S, Poonpolsup S, et al. New chapter in tackling antimicrobial resistance in Thailand. BMJ 2017;358.

30. Pumart $P$, Prakongsai $P$, Thamlikitkul $P$, et al. Health and economic impacts of antimicrobial resistant infections in Thailand: a preliminary study. J Health Serv Res Pol 2012;6:352-60.

31. Review WP. Thailand population 2017, 2017. Available: http://worl dpopulationreview.com/countries/thailand-population/ [Accessed 5 Jan 2018].

32. Van Boeckel TP, Brower C, Gilbert M, et al. Global trends in antimicrobial use in food animals. Proc Natl Acad Sci U S A 2015;112:5649-54 
33. Food and Agriculture Organization of the United Nations. Proceedings of the International Workshop on the use of antimicrobials in livestock production and antimicrobial resistance in the Asia-Pacific region, 2012. Available: http://cdn.aphca.org/ dmdocuments/AMR WS Proceedings_121112.pdf

34. The World Bank. Sri Lanka, 2017. Available: http://data.worldbank. org/country/sri-lanka [Accessed 5 Jan 2018].

35. US Poultry \& Egg Association. Economic data, 2015. Available: https://www.uspoultry.org/economic_data/ [Accessed 20 Oct 2015].

36. Center for Disease Dynamics EP. Antibiotic use in Sri Lanka, 2017. Available: https://resistancemap.cddep.org/CountryPage.php? countryld=100\&country=Sri+Lanka [Accessed 24 Nov 2017].

37. England PH. English Surveillance Programme for Antimicrobial Utilisation and Resistance (ESPAUR): report 2014. London, England; 2014.

38. Public Health Agency of Sweden NVI. Consumption of antibiotics and occurrence of antibiotic resistance in Sweden. Solna and Uppsala, Sweden; 2015.

39. PwC. The health sector of Sri Lanka, 2014. Available: https://www. rvo.nl/sites/default/files/2016/01/Health sector in Sri Lanka.pdf [Accessed 24 Nov 2017].

40. Dharmaratne S, Agampodi S, Dassanayaka S, et al. Disease burden assessment beyond in-patient data: a morbidity profile assessment of outpatients. Int J Prev Med 2012;3:730-2.

41. Statistica. Sri Lanka: inflation rate from 2012 to 2022, 2018. Available: https://www.statista.com/statistics/728516/inflation-ratein-sri-lanka/ [Accessed 5 Jan 2018].

42. You JHS, Tam L-P, Lee NLS. Cost-effectiveness of molecular pointof-care testing for influenza viruses in elderly patients at ambulatory care setting. PLoS One 2017;12:e0182091.

43. Dugas AF, Coleman S, Gaydos CA, et al. Cost-utility of rapid polymerase chain reaction-based influenza testing for high-risk emergency department patients. Ann Emerg Med 2013;62:80-8.

44. Shen K, Xiong T, Tan SC, et al. Oseltamivir treatment for children with influenza-like illness in China: a cost-effectiveness analysis. PLoS One 2016;11:e0153664.

45. Lavelle TA, Uyeki TM, Prosser LA. Cost-effectiveness of oseltamivir treatment for children with uncomplicated seasonal influenza. $J$ Pediatr 2012;160:e66:67-73.

46. Lubell Y, Do NTT, Nguyen KV, et al. C-reactive protein point of care testing in the management of acute respiratory infections in the Vietnamese primary healthcare setting - a cost benefit analysis. Antimicrob Resist Infect Control 2018;7.

47. Shrestha P, Cooper BS, Coast J, et al. Enumerating the economic cost of antimicrobial resistance per antibiotic consumed to inform the evaluation of interventions affecting their use. Antimicrob Resist Infect Control 2018;7.

48. The Review on Antimicrobial Resistance. Antimicrobial resistance: tackling a crisis for the health and wealth of nations; 2014.

49. ARSP Working Group, The Sri Lanka College of Microbiologists. A multi centre laboratory study of gram negative bacterial blood stream infections in Sri Lanka. Ceylon Med J 2013;58:56-61. Jun.

50. Wickramatilake CM, Dharmadasa RA, De Silva M. Are we over prescribing antibiotics in children with acute lower respiratory tract infections in Sri Lanka? Acta Paediatr 2017;106.

51. Oppong R, Smith RD, Little $P$, et al. Cost effectiveness of amoxicillin for lower respiratory tract infections in primary care: an economic evaluation accounting for the cost of antimicrobial resistance. $\mathrm{Br} \mathrm{J}$ Gen Pract 2016;66:e633-9.

52. Drain PK, Hyle EP, Noubary F, et al. Diagnostic point-of-care tests in resource-limited settings. Lancet Infect Dis 2014;14:239-49.

53. Nshimyumukiza L, Douville X, Fournier D, et al. Cost-effectiveness analysis of antiviral treatment in the management of seasonal influenza A: point-of-care rapid test versus clinical judgment. Influenza Other Respir Viruses 2016;10:113-21.

54. Montalto NJ. An office-based approach to influenza: clinical diagnosis and laboratory testing. Am Fam Physician 2003;67:111-8.

55. Esposito S, Marchisio P, Morelli P, et al. Effect of a rapid influenza diagnosis. Arch Dis Child 2003;88:525-6.

56. Bonner AB, Monroe KW, Talley LI, et al. Impact of the rapid diagnosis of influenza on physician decision-making and patient management in the pediatric emergency department: results of a randomized, prospective, controlled trial. Pediatrics 2003;112:363-7.

57. Huang DT, Yealy DM, Filbin MR, et al. Procalcitonin-Guided use of antibiotics for lower respiratory tract infection. $N$ Engl J Med 2018;379:236-49.

58. Briel M, Schuetz P, Mueller B, et al. Procalcitonin-guided antibiotic use vs a standard approach for acute respiratory tract infections in primary care. Arch Intern Med 2008;168:2000-7.

59. Burkhardt $\mathrm{O}$, Ewig S, Haagen U, et al. Procalcitonin guidance and reduction of antibiotic use in acute respiratory tract infection. Eur Respir J 2010;36:601-7.

60. Yang L, Liu C, Wang L, et al. Public reporting improves antibiotic prescribing for upper respiratory tract infections in primary care: a matched-pair cluster-randomized trial in China. Health Res Policy Syst 2014;12

61. Yip W, Powell-Jackson T, Chen W, et al. Capitation combined with pay-for-performance improves antibiotic prescribing practices in rural China. Health Aff 2014;33:502-10.

62. Kazungu JS, Barasa EW, Obadha M et al. What characteristics of provider payment mechanisms influence health care providers' behaviour? A literature review. Int J Health Plann Manage 2018;33:e892-905

63. Thorpe KE, Joski P, Johnston KJ. Antibiotic-Resistant Infection Treatment Costs Have Doubled Since 2002, Now Exceeding \$2 Billion Annually. Health Aff 2018;37:662-9.

64. Goossens H, Ferech M, Vander Stichele R, et al. Outpatient antibiotic use in Europe and association with resistance: a crossnational database study. Lancet 2005;365:579-87.

65. van de Sande-Bruinsma N, Grundmann H, Verloo D, et al. Antimicrobial drug use and resistance in Europe. Emerg Infect Dis 2008;14:1722-30.

66. Albrich WC, Monnet DL, Harbarth S. Antibiotic selection pressure and resistance in Streptococcus pneumoniae and Streptococcus pyogenes. Emerg Infect Dis 2004;10:514-7.

67. The World Bank. Ppp conversion factor, GDP (LCU per international \$), 2015. Available: http://data.worldbank.org/indicator/PA.NUS.PPP [Accessed 23 Nov 2017].

68. The World Bank. United States, 2017. Available: http://data worldbank.org/country/united-states [Accessed 23 Nov, 2017].

69. XE. Current and historical rate tables, 2017. Available: http://www. xe.com/currencytables/?from=USD\&date=2013-01-01 [Accessed 23 Nov 2017]. 